\title{
Usefulness of therapeutic drug monitoring of piperacillin and meropenem in routine clinical practice: a prospective cohort study in critically ill patients
}

\author{
Joan Antoni Schoenenberger-Arnaiz 지, , ${ }^{1}$ Faten Ahmad-Diaz, ${ }_{1}^{2}$ Mar Miralbes-Torner, ${ }^{3}$ \\ Ana Aragones-Eroles, ${ }^{2}$ Manuel Cano-Marron, ${ }^{1}$ Mercedes Palomar-Martinez ${ }^{3}$
}

\begin{abstract}
- Additional material is published online only. To view please visit the journal online (http://dx.doi.org/ 10.1136/ejhpharm-2018001713).
\end{abstract}

${ }^{1}$ Pharmacy, Hospital Universitari Arnau de Vilanova, Lleida, Spain

Intensive Care Unit, Hospital Universitari Arnau de Vilanova, Lleida, Spain

${ }^{3}$ Pharmacoepidemiology, Institut de Recerca Biomedica de Lleida, Lleida, Spain

Correspondence to

Dr Joan Antoni

Schoenenberger-Arnaiz,

Pharmacy, Hospital Universitari

Arnau de Vilanova, Lleida

25198, Spain; jas.Ileida.ics@ gencat.cat

Received 4 August 2018 Revised 27 December 2018 Accepted 5 February 2019 Published Online First 27 February 2019

EAHP Statement 4: Clinical Pharmacy Services.

\section{ABSTRACT \\ Background Beta-lactam anti-infective levels after} standard dosing have been shown to be subtherapeutic when renal clearance is augmented.

Objective To determine if piperacillin and meropenem are found to be in their therapeutic range in infected critically ill patients when administered by continuous intravenous infusion (CII) assisted by a therapeutic drug monitoring (TDM) report issued by the pharmacy service. Methods This prospective non-controlled intervention study evaluated septic patients in an intensive care unit. Patients received a loading dose of meropenem or piperacillin-tazobactam and the antibiotics were afterwards administered by CII. Blood concentrations were determined by high-performance liquid chromatography assays. The adequacy of $\beta$-lactam therapy in the cohort subjected to intervention was assessed by determining whether plasma levels during Cll were $>4$ times the informed minimum inhibitory concentration during the first 96 hours of treatment. Results A total of 124 patients were subject to TDM during antibiotic treatment but, for the analysis of the fulfilment of pharmacodynamic requirements, data from $31 / 124(25 \%)$ were excluded. Of the whole cohort of treatment courses, $57 / 93(61.3 \%)$ reached the target level. Plasma levels were adequate in 41/70 (58.6\%) and $16 / 23(69.6 \%)$ of the patients treated with piperacillintazobactam and meropenem, respectively. Globally, recommendations based on TDM results were followed in $35 / 93(37.6 \%)$ of the treatment courses.

Conclusions The results of the study show that, in critically ill patients with sepsis, there is a significant proportion of treatment courses where target levels are not reached even if the antibiotics are administered by CII and TDM support is provided by the pharmacy service. This TDM support should be offered on a real-time basis to be really useful.

\section{INTRODUCTION}

(C) European Association of Hospital Pharmacists 2020. No commercial re-use. See rights and permissions. Published by BMJ.

To cite: SchoenenbergerArnaiz JA, Ahmad-Diaz F, Miralbes-Torner $\mathrm{M}$, et al. Eur J Hosp Pharm 2020;27:e30-e35.
Severe infections remain a major challenge for hospital medicine and make it necessary to explore new ways to maximise effectiveness and safety when managing available antibiotics. With this objective in mind, application of knowledge of the pharmacokinetic (PK) and pharmacodynamic (PD) profiles for these drugs in clinical practice is critical. ${ }^{12}$

Pharmacodynamically, $\beta$-lactams are timedependent antibiotics. Their activity is related to the time during which the antibiotic concentration remains above four or five times the minimum inhibitory concentration (MIC) for a particular microorganism, and this time (fT $>$ MIC) should be $30-70 \%$ of the dosing interval. ${ }^{13}$ However, the pharmacokinetics of $\beta$-lactams can be highly variable depending on the clinical condition of the patient, leading to lower than expected drug concentrations of these drugs in critically ill patients. ${ }^{45}$ In turn, this can reduce the time that the antibiotic concentration remains above the MIC, requiring dose adjustment. ${ }^{4}$ The consequences of inadequate antibiotic exposure are the emergence of antibiotic resistance ${ }^{67}$ and ineffectiveness in eradicating the infection, which can increase the length of hospital stays and decrease the quality of patient recovery or even survival. ${ }^{4}$

The measurement of plasma concentrations of antimicrobials and their comparison with the MIC of the causative organism of infection is an effective tool to individualise treatment, increase clinical efficacy and reduce the selection of resistant mutants. ${ }^{4}$ This tool is known as the pharmacokinetic/pharmacodynamic (PK/PD) guided dosage. On the other hand, some adverse effects such as encephalopathy and seizures occur when high concentrations of these drugs are reached. The situation is more probable in infections requiring higher doses of antibiotic, and determining the plasma concentrations of $\beta$-lactams can help optimise treatment by limiting the risk of dose-related adverse effects and avoiding suboptimal concentrations. ${ }^{89}$ Meropenem (MEM) and piperacillin-tazobactam (PTZ) are of great interest for therapeutic drug monitoring (TDM) because of their wide use in critically ill patients. ${ }^{10}$

Previous studies have shown that continuous intravenous administration (CII) of $\beta$-lactam antibiotics in severe sepsis may help overcome subtherapeutic PTZ concentrations and even increase rates of clinical cure. ${ }^{11-14}$ The aim of this study was to investigate the usefulness of a TDM report provided by the pharmacy service to fulfil the pharmacodynamic requirements for MEM and PTZ in this population of patients when these antibiotics are administered by CII. In addition, this study sought to determine the PK parameters in the studied population for both antibiotics and to identify factors that influence the achievement of the targeted values for PK/ PD indexes. 
MATERIALS AND METHODS

\section{Study design, patients and anti-infective treatments}

This was a prospective non-controlled interventional study performed in an intensive care unit (ICU) in a University Hospital. The study protocol was approved by the Ethical Committee of Investigation from the hospital and, before enrollment, written consent was obtained from the patients or their relatives. Inclusion criteria were a diagnosis of severe sepsis or septic shock, ${ }^{15}$ either at admission or during the ICU stay, and the need for treatment either with PTZ or MEM. Patients meeting one of the following criteria were not eligible for the study: age $<18$ years; pregnancy or lactation; previous administration of any of the researched antibiotics; allergy to any of the investigated antibiotics; diagnosis of meningitis or cystic fibrosis; inability to answer questions or a lack of relatives; inclusion in a clinical trial in the previous 6 months; or dementia. The study period was limited to the first 96 hours of antibiotic therapy, with a minimum observation period of 72 hours.

Once enrolled, patients starting treatment with MEM in the ICU received a loading dose of 1 or $2 \mathrm{~g}$ administered over 30 min and immediately after its completion they received a CII of 3 g or 6 g every 24 hours. For stability reasons, piggy-bags were changed every 8 hours. Patients starting treatment with PTZ in the ICU received a loading dose of 4-0.5 g administered over $30 \mathrm{~min}$, followed immediately by a CII of $16-2 \mathrm{~g}$ or $24-3 \mathrm{~g}$ every 24 hours. In patients in whom the anti-infective treatment was initiated before ICU admission, the CII protocol was directly applied. When needed, continuous renal replacement therapy (CRRT) was given. Veno-venous haemodialysis (CVVHD) was performed using a Prismaflex System with either a polyacrylonitrile (AN69) Prismaflex M100 (0.9 $\mathrm{m}^{2}$ membrane surface area) or an AN69 Prismaflex M150 (1.5 $\mathrm{m}^{2}$ membrane surface area). The vast majority of patients were re-infused with pre-dilution and the usual doses used were $35 \mathrm{~mL} / \mathrm{kg} /$ hour of effluent and 20 $\mathrm{mL} / \mathrm{kg} /$ hour of ultrafiltrate.

\section{Interventions}

For the purpose of the study, an intervention was defined as the determination of the antibiotic plasma level of either of the two antibiotics accompanied by a written report from the Pharmacy Service. On the request of the ICU attending physician via the electronic clinical workstation, the Pharmacy Service performed the analytical determination and, for both antibiotics and with each plasma level determination, attending physicians received a report from the Pharmacy Department indicating the PK/PD index if microbiological data were available. The report included indications for dose changes if this was necessary; those changes were based on the calculated total clearance (CL) of the drug and the target serum concentration at steady state (Css). If there was a change in renal function and if the TDM report was not available, the dose adjustment was done according to existing official technical brochures. ${ }^{1617}$

\section{Analysis of meropenem and piperacillin plasma levels}

Blood samples were collected $30 \mathrm{~min}$ after the infusion of the loading dose and every 24 hours during the consecutive continuous infusion until the 96-hour study period had expired. These blood-draw time points were chosen as maximal serum concentration (Cmax) and Css, respectively. However, although $\beta$-lactam levels were run in-house, the TDM programme runs drug assays twice weekly and thus the results were only available twice a week
Importantly, as the PK of piperacillin and tazobactam are highly correlated, ${ }^{18}$ only piperacillin levels were measured. The free drug concentration for piperacillin was calculated considering piperacillin protein binding (30\%). Serum $\beta$-lactam concentrations were measured by high-performance liquid chromatography (with diode array detection. ${ }^{18}$ Details of the analytical methods for piperacillin and meropenem are given in online supplementary file 1.

\section{PK analysis and PK/PD endpoints}

PK parameters researched included Cmax, volume of distribution (Vd), Css, CL and area under the serum concentration-time curve (AUC). Vd and CL were normalised to body weight.

The fulfilment of PK/PD $\beta$-lactam endpoints was assessed by determining if plasma levels during continuous infusion were $>4$ times MIC. Two different situations could occur. For the pathogens of interest, the MIC determination was either established by our microbiology laboratory or, in the absence of this information, an empirical target threshold was set considering the MIC for resistant pathogens (such as Pseudomonas aeruginosa, commonly isolated in ICU patients) usually indicated by our microbiological laboratory; in this case the highest MIC in the susceptible range was selected as the dosing target: $\leq 16 \mu \mathrm{g} /$ $\mathrm{mL}$ for PTZ and $\leq 4 \mu \mathrm{g} / \mathrm{mL}$ for MEM. ${ }^{19}$ If all Css determinations performed in the first 96 hours were $>4$ times MIC, this was considered as a PK/PD index of $100 \%$. Broth microdilution was used to test strain susceptibility and the E-test was performed as confirmation if considered. As a rule of thumb, to obtain a proper $\mathrm{PK} / \mathrm{PD}$ result the E-test was used when the plasma concentrations obtained were considered low and bacteria susceptibility was $<1 \mu \mathrm{g} / \mathrm{ml}$ for MEM or $<16 \mu \mathrm{g} / \mathrm{ml}$ for piperacillin. The early $\mathrm{Vd}$ of both antibiotics was calculated using the measured plasma antibiotic concentration determined $30 \mathrm{~min}$ after the administration of a loading dose. This value was considered a Cmax and the following equation was applied: $\mathrm{Vd}(\mathrm{L} / \mathrm{kg})=$ [loading dose $(\mathrm{mg}) / \mathrm{Cmax}(\mathrm{mg}(\mathrm{L})] /$ patient weight $(\mathrm{kg})$.

Clearance of PTZ and MEM was calculated for each measured plasma antibiotic concentration during the 96-hour period of study including the values for Css and the corresponding dosing rate (DR) of the drug in the following equation: CL $(\mathrm{L} / \mathrm{h})=\mathrm{DR}$ $(\mathrm{mg} / \mathrm{h}) / \mathrm{Css}(\mathrm{mg} / \mathrm{L})$. For each treatment course several Css values could be measured. For every measurement obtained, the corresponding drug CL was determined in relation to the dose administered. The TDM report to the physician may include a recommended dose rate change based on calculated drug $\mathrm{CL}$ and target Css, applying the same equation. For each CL determined, the AUC expressed in $\mu \mathrm{g}$ *hour $/ \mathrm{mL}$ was estimated using the formula $\mathrm{AUC}=$ dose/CL (with dose expressed in $\mathrm{mg} /$ hour and $\mathrm{CL}$ in $\mathrm{mL} /$ hour). Following this, all $\mathrm{CL}$ and AUC values were pooled to establish the mean and SD.

\section{Data management and statistical analysis}

Demographics, previous chronic diseases, admission diagnosis and biochemistry results were collected from institutional electronic records. The severity of disease for each patient was characterised using the Acute Physiology and Chronic Health Evaluation (APACHE) II. ${ }^{20}$ Creatinine clearance was calculated using a standard formula. ${ }^{21}$ Details of the treatment with CRRT were recorded, as were length of ICU and hospital stay, overall mortality and cause of death.

Data including drug treatment and the abovementioned variables were registered for each course of treatment (episode) along with Cmax and Css determinations. For MEM, patients 


\begin{tabular}{ll}
\hline $\begin{array}{l}\text { Table } 1 \quad \text { Patient demographic and haemodynamic data on admission } \\
\text { to ICU }(\mathrm{n}=124)\end{array}$ \\
\hline Characteristics \\
\hline Mean age (years) \\
\hline Male/female & $61.5 \pm 15.0$ \\
\hline Body mass index & $89 / 35$ \\
\hline APACHE II on admission & $30.2 \pm 6.6$ \\
\hline Comorbidities & $18.1 \pm 7.5$ \\
\hline \multicolumn{1}{c}{ Diabetes } & \\
\hline COPD & $31(25 \%)$ \\
\hline Chronic renal failure & $25(20.2 \%)$ \\
\hline Malnutrition & $17(13.7 \%)$ \\
\hline Immunosuppression & $17(13.7 \%)$ \\
\hline Malignancy & $21(16.9 \%)$ \\
\hline Liver cirrhosis & $46(37.1 \%)$ \\
\hline Bacteraemia & $13(10.5 \%)$ \\
\hline Septic shock & $25(20.2 \%)$ \\
\hline & $77(62 \%)$ \\
\hline
\end{tabular}

with a glomerular filtration rate (GFR) $<50 \mathrm{~mL} / \mathrm{min}$ at any time during the 96-hour study period were classified as needing dose reduction. ${ }^{16}$ In the case of PTZ, this value was fixed at $40 \mathrm{~mL} / \mathrm{min} .{ }^{17}$ For the analysis of $\mathrm{PK}$ parameters and PK/PD indexes, treatment courses were divided into three groups: (A) dose reduction not required and no CRRT; (B) dose reduction required and no CCRT; $(\mathrm{C})$ with CRRT at any time during the observation period.

Differences in continuous variables between patients in different clinical situations were analysed using the one-way analysis of variance (ANOVA) test. Differences in qualitative variables were analysed using the $\chi^{2}$ test. The $95 \%$ CI was determined for the observed proportions and associations were expressed as relative risk ratios with $95 \%$ confidence intervals. A two-tailed $p$ value of 0.05 was considered statistically significant and all analyses were performed using Version 17.0 of the SPSS software package (SPSS, Chicago, Illinois, USA).

\section{RESULTS}

\section{Patient characteristics}

The total study cohort included 124 patients (table 1). During the stay in ICU, 58 (47\%) developed renal failure and 34 $(27.4 \%)$ received haemofiltration. Treatment with mechanical ventilation was initiated in 80 patients (64.5\%). Overall ICU mortality among study patients, considering the whole stay, was $12.9 \%$. There was no difference in mortality between patients who reached the target PD antibiotic levels and those who did not. Among patients in whom the dose was changed (mostly reduced), the all-cause mortality rate was 9/36 (25\%) compared with $8 / 57(10.5 \%)$ among those in whom there were no changes in dose. However, in the five patients whose death was deemed directly related to infection, the therapeutic range was achieved.

\section{Dose schedules and PK/PD indexes}

Most patients received loading doses of PTZ or MEM (87/92 and $28 / 32$, respectively) and continued afterwards with high doses of the antibiotic (62/92 with 24-3 g daily for PTZ and 29/32 with $6 \mathrm{~g}$ daily for MEM). For the analysis of the fulfilment of PD requirements, data from 31/124 (25\%) were excluded due to the following causes: in 13 cases MEM or PTZ was discontinued or switched to another anti-infective because of the microbiology department recommendations after obtaining the results for the cultures and 18 patients were discharged from the ICU
Table 2 Distribution of treatment courses by antibiotic and number of courses with serum concentration at steady state (Css) above the established target for each group. A: dose reduction not required and no continuous renal replacement therapy (CRRT). B: dose reduction required and no CCRT. C: CRRT at any time during the observation period

\begin{tabular}{|c|c|c|}
\hline Antibiotic & $\begin{array}{l}\text { Treatment courses } \\
\mathrm{n} \text { ( } \% \text { of drug cases) }\end{array}$ & $\begin{array}{l}\text { Courses with all } \\
\text { Css above target } \\
\text { during } 96 \text { hours } \\
\text { n (\% of group) }\end{array}$ \\
\hline \multirow{5}{*}{$\begin{array}{l}\text { Piperacillin- } \\
\text { tazobactam (PTZ) }\end{array}$} & Global $70(75.0)$ & 41 (58.6) \\
\hline & Group A, n (\% PTZ) 29 (40.0) & $9(31.0)$ \\
\hline & Groups B and C, n (\% PTZ) 41 (63.6) & $32(78.0)$ \\
\hline & $\begin{array}{l}\text { Specific therapy } \mathbf{n}(\% \mathrm{PTZ}) \\
\text { E. coli } 13 \\
\text { P. aeruginosa } 7 \\
\text { Other } 5 \\
\text { All } 25(37.9)\end{array}$ & $15(60.0)$ \\
\hline & Empirical therapy n (\% PTZ) 45 (64.3) & $26(57.8)$ \\
\hline \multirow[t]{5}{*}{ Meropenem (MEM) } & Global $23^{25}$ & $16(69.6)$ \\
\hline & Group A, n (\% MEM) 14 (60.9) & $7(50.0)$ \\
\hline & Group B and C, n (\% MEM) 9 (39.1) & $9(100)$ \\
\hline & $\begin{array}{l}\text { Specific therapy, } \mathrm{n} \text { (\% MEM) } \\
\text { E. coli } 12 \\
\text { P. aeruginosa } 1 \\
\text { Other } 1 \\
\text { All } 14(60.9)\end{array}$ & $13(92.8)$ \\
\hline & Empirical therapy, $n$ (\% MEM) 9 (39.1) & $3(33.3)$ \\
\hline Total & $93(100)$ & $57(61.3)$ \\
\hline
\end{tabular}

before completion of the minimum 72-hour observation period. It is important to note that, in $49 / 93(52.6 \%)$ of the treatment courses, only one Css value was available. For the others, two or three determinations were available in $41 / 93$ patients $(44.1 \%)$ and $3 / 93$ patients $(3.3 \%)$, respectively.

As shown in table 2, most patients received PTZ. For the whole cohort, the proportion of courses in which the target level was not reached is high $(36 / 93 ; 38.7 \%)$. However, $6 / 36$ patients who were deemed subtherapeutic (because not all their Css levels were above target during 96 hours) had therapeutic levels at the time of the second TDM when the first TDM had been subtherapeutic. For patients with normal renal function and no need for haemofiltration (group A), the proportion of courses not reaching the target level was higher $(27 / 43 ; 62.7 \%)$. Furthermore, for these patients the risk of having Css levels under target was higher with PTZ than with MEM, although the difference was not statically significant. In contrast, when the treatment courses of patients with renal failure or undergoing haemofiltration (groups B and C) were analysed, it was observed that there were fewer courses where target levels were not reached.

\section{Dose adjustments and response to intervention}

Dose adjustment in the cohort of patients selected with the aim of analysing PD requirements fulfilment was made in 36/93 (38.7\%) of treatment courses. In most cases the dose was reduced and only in four cases (11.1\% of changes) was the dose increased. Doses were adjusted less frequently with MEM than with PTZ: $2 / 23(8.7 \%)$ vs 34/71 $(47.8 \%)$, respectively $(\mathrm{p}<0.01)$. For the group of patients that were not receiving high doses (11 patients) and for whom target levels were not being reached, in only one case was the dose increased whereas the dose was maintained or even reduced in the remainder. Furthermore, the dose was reduced in 6/33 patients who were receiving high doses 


\begin{tabular}{|c|c|c|c|c|c|c|}
\hline & $\begin{array}{l}\text { Css }(m g / l) \\
\text { Mean } \pm S D\end{array}$ & $\begin{array}{l}\text { P value } \\
\text { (ANOVA) }\end{array}$ & $\begin{array}{l}\mathrm{CL}(\mathrm{ml} / \mathrm{kg} \cdot \mathrm{min}) \\
\text { Mean } \pm \mathrm{SD}\end{array}$ & $\begin{array}{l}\text { P value } \\
\text { (ANOVA) }\end{array}$ & $\begin{array}{l}\text { AUC }(\mu \mathrm{g} \cdot \mathrm{h} / \mathrm{ml}) \\
\text { Mean } \pm \text { SD }\end{array}$ & $\begin{array}{l}\text { P value } \\
\text { (ANOVA) }\end{array}$ \\
\hline \multicolumn{7}{|l|}{ Meropenem ( $\mathrm{n}=$ Css determinations) } \\
\hline (A) Dose adjustment not required and no CRRT ( $\mathrm{n}=26$ ) & $20.7 \pm 10.3$ & $<0.0001$ & $3.2 \pm 1.6$ & $<0.0001^{*}$ & $74.6 \pm 37.9$ & $<0.0001$ \\
\hline (B) Dose adjustment required and no CRRT ( $\mathrm{n}=6$ ) & $65.5 \pm 25.4$ & & $0.8 \pm 0.3$ & & $234.3 \pm 91.4$ & \\
\hline (C) With CRRT $(n=9)$ & $39.8 \pm 16.4$ & & $1.18 \pm 0.3$ & & $139.8 \pm 59.1$ & \\
\hline All cases $(n=41)$ & $30.8 \pm 21.4$ & & $2.43 \pm 1.7$ & & $110.8 \pm 76.9$ & \\
\hline \multicolumn{7}{|l|}{ Piperacillin } \\
\hline (A) Dose adjustment not required and no CRRT ( $\mathrm{n}=63$ ) & $72.5 \pm 46.8$ & $<0.0001 \dagger$ & $3.8 \pm 2.6$ & $<0.0001 \dagger$ & $261.0 \pm 168.4$ & $<0.0001 \dagger$ \\
\hline (B) Dose adjustment required and no CRRT ( $\mathrm{n}=24)$ & $138.6 \pm 44.5$ & & $1.1 \pm 0.5$ & & $499.1 \pm 160.1$ & \\
\hline (C) With CRRT $(n=40)$ & $138.4 \pm 57.9$ & & $1.2 \pm 0.6$ & & $498.2 \pm 208.6$ & \\
\hline All cases $(n=127)$ & $108.8 \pm 59.0$ & & $2,5 \pm 2.0$ & & $380.0 \pm 212.5$ & \\
\hline
\end{tabular}

${ }^{*}$ Differences not statistically significant between groups B and C.

tDifferences not statistically significant between groups $B$ and $C$.

AUC, area under the curve; $\mathrm{CL}$, clearance; CRRT, continuous renal replacement therapy; Css, serum concentration at steady state.

but also did not reach target levels. Thus, recommendations to increase the dose based on TDM results were accepted by the medical staff in 5/36 (13.8\%) of courses and globally recommendations based on TDM results were followed in 35/93 (37.6\%) of the courses.

\section{Pharmacokinetic data}

All treatment courses were included in the analysis of CL rates and AUC with no exclusions (lack of at least one Css level 24-96 hours from the beginning of the treatment was a criterion for exclusion). The mean PK parameters for the two drugs are shown in table 3. There was marked interindividual variation in all PK parameters. For MEM, data were available to estimate the early $\mathrm{Vd}$ in 12 patients. The mean \pm SD for MEM Vd was $0.40 \pm 0.2 \mathrm{~L} /$ $\mathrm{kg}$ and the Cmax for free drug after a $2 \mathrm{~g}$ dose was $52.9 \pm 24.6$ $\mu \mathrm{g} / \mathrm{mL}$. For PTZ, data were available for estimating the $\mathrm{Vd}$ in 30 patients. The mean \pm SD for PTZ Vd was $0.68 \pm 0.26 \mathrm{~L} / \mathrm{kg}$ and the Cmax for free drug after a $4 \mathrm{~g}$ dose was $96.5 \pm 22.3 \mu \mathrm{g} / \mathrm{mL}$.

Patients treated with MEM, with GFR $>50 \mathrm{~mL} /$ hour and not undergoing CRRT (group A) received a mean \pm SD dose of $243.8 \pm 37.8 \mathrm{mg} /$ hour while those in groups B and C received $234.4 \pm 44.2$ and $218.7 \pm 57.9 \mathrm{mg} /$ hour, respectively. Patients with normal renal function and not undergoing haemofiltration had lower Css and AUC values than patients with renal failure. In accordance, the former had higher CL rates than the latter. Patients undergoing haemofiltration had intermediate values.

Patients treated with PTZ, with GFR $>40 \mathrm{~mL} /$ hour and not undergoing CRRT (group A) received a mean \pm SD dose of $947.6 \pm 191.0 \mathrm{mg} /$ hour. Patients in group C received $754.2 \pm 244.6 \mathrm{mg} /$ hour, while those in group B received $780.0 \pm 244.0 \mathrm{mg} /$ hour. Differences between groups A and B and $\mathrm{B}$ and $\mathrm{C}$ were statistically significant, but not between groups $\mathrm{B}$ and C. For MEM, patients in group A had lower Css and AUC values than patients needing per protocol dose adjustment (group B) despite receiving higher doses; also, the former had higher CL rates than the latter. In contrast with what happened in patients treated with MEM, patients treated with PTZ and submitted to haemofiltration (group C) had similar Css, CL and AUC to patients in group B while receiving similar doses.

\section{DISCUSSION}

The results of the present study indicate that, in our clinical practice and the cohort of patients analysed, the usefulness of TDM to increase the degree of fulfilment of PD requirements for MEM and PTZ is limited. In the present study, the TDM-based recommendations to increase or maintain the dose (once the plasma level was available) were not followed in more than a third of cases. The poor response of ICU physicians to these recommendations partly explains the low index of coverage of PD requirements, especially in the case of PTZ. These results contrast with recently published work indicating that clinical pharmacological advice may enable very high adherence rates by clinicians to dosing recommendations based on TDM. ${ }^{22}$ Although the reasons for accepting the suggestions or not were not registered during the study, this poor response can be attributed to the fact that there is still inadequate knowledge regarding the value of PK/PD indexes in the use of $\beta$-lactam antibiotics and would suggest the need for future training interventions. The availability of only one determination for the 72-96 hour treatment course in a majority of patients could have additionally jeopardised the usefulness of TDM. In fact, the availability of PK advice in real time appears to be decisive for the success of these interventions. Han et al demonstrated that 24/7 PK services implemented with institutional Pharmacy Practice Model expansion significantly improved vancomycin dosing when compared with a previous phase that included universal monitoring by pharmacists with recommendations made to prescribers only during business hours. ${ }^{23}$

Previously published reviews on this topic suggest that continuous infusion of $\beta$-lactam antibiotics may have variable efficacy in different patient groups, ${ }^{24}$ and that this efficacy could be related to the amount of time that the unbound fraction of antibiotic remains above the MIC of the pathogens. ${ }^{25}$ Patients who may benefit from CII are critically ill patients with very severe illnesses. ${ }^{26}$ To the best of our knowledge, this is the first study in which the TDM technique has been used to optimise treatment with intravenous continuous PTZ infusions in routine clinical practice. TDM of PTZ in critically ill patients receiving CII has been reported previously, ${ }^{27} 28$ but in these communications there was no reference to dose adjustments based on the measured PTZ serum levels. The effect of TDM-assisted optimisation of high-dose continuous MEM infusions on the clinical outcome of seriously infected patients has been previously reported. ${ }^{29}$ Our data show that a high proportion of critically ill patients submitted to this modality of treatment for PTZ or MEM do not attain the targeted plasma drug level. Others have obtained similar results in patients receiving these antibiotics by intermittent short intravenous infusions or continuous infusions. ${ }^{29-31} \mathrm{~A}$ previous clinical trial showed that a strategy of dose adaptation based on daily TDM led to an increase in 
PK/PD target attainment compared with conventional dosing ${ }^{31}$ ; this study included Critically Ill Patients (CIP) with normal kidney function that received extended infusions of MEM or PTZ and found that, at 72 hours, patients in the intervention group had 58\% target attainment rates for $100 \%$ fT $>4 \times$ MIC . In contrast, as has also been previously demonstrated, patients with renal failure or undergoing haemofiltration easily fulfil the PD requirements for both antibiotics. ${ }^{30} 32$

We have chosen an aggressive approach targeting 100\% fT $>4 \times$ MIC levels by using high doses of the antibiotics in CII. When high doses are used to treat less susceptible strains, overdosing and toxicity of $\beta$-lactams could also be of concern, so drug monitoring would also help in this situation to correctly adjust the dose. ${ }^{33}$ Other authors have targeted similar PD indexes and observed that dose increases were needed in many patients. ${ }^{34}$

Our study also confirms the dramatic changes induced by sepsis in the PK parameters for the studied antibiotics, especially the $\mathrm{Vd}$, as demonstrated in previous published work. ${ }^{35}$ The values we found for the early Vd of MEM and PTZ are higher than those reported in population pharmacokinetic studies ${ }^{36-38}$ where more sophisticated methods to estimate PK parameters have been used. In our study we have used the Aardema et al approach, ${ }^{28}$ where the first hour of starting treatment, including infusion of the loading dose directly followed by continuous infusion, was considered the loading phase. Thus the concentration measured at the end of this loading phase was deemed a true Cmax. The same approach has been used for MEM as a Tmax of 1 hour has been reported previously. ${ }^{39}$ Published models suggest that loading doses (LDs) may be necessary for the modelled PTZ CII schemes and for elevated MICs. ${ }^{40}$ Drug clearance remains the main determinant for drug concentrations at steady state and, as described earlier, critically ill patients with normal renal function show augmented renal clearance for $\beta$-lactam antibiotics. While normal doses of PTZ and MEM could be sufficient to treat strains susceptible to these drugs, for less susceptible pathogens a higher than recommended regimen using CII would be necessary to optimise the efficacy of these antibiotics.

In patients receiving PTZ, although the $1.2 \mathrm{~mL} / \mathrm{kg}$. min clearance values we found in patients with CVVHF are in line with those described in the available literature, ${ }^{32} 41$ it appears that the renal replacement therapy (RRT) system used did not improve piperacillin clearance. In patients receiving PTZ CIIs, our results do not support the suggestions to use higher doses of this antibiotic in patients with CVVHF than in patients with renal failure who are not undergoing RRT. ${ }^{41}$

Previous PD data for MEM in patients with ventilatorassociated pneumonia following administration by 3-hour infusion indicate AUC values well above those found in the present study for similar doses. ${ }^{42}$ However, in the referenced study patients had a much lower mean body weight than our population and, in addition, the cohort did not include patients with septic shock. The proposed CII rates of MEM in septic patients with different degrees of renal function that theoretically allow achievement of plasma drug concentrations above $16 \mathrm{mg} / \mathrm{L}$ are under the values that we found. ${ }^{43}$ In contrast with what we found for PTZ and as a result of higher clearance rates of MEM in patients with RRT, an increase in dosage should be faced when this technique is initiated. ${ }^{44}$

The overall ICU mortality registered in the present study was low compared with other cohorts of septic critically ill patients, ${ }^{34}$ but similar or even higher than that found by other investigators using antibiotic extended infusion strategies ${ }^{11}$ or TDM-driven dose optimisation. ${ }^{29}$
What this paper adds

What is already known on this subject

- In critically ill patients there is a high variability in the blood $\beta$-lactam antibiotic levels achieved with continuous intravenous infusion of the drug.

- In critically ill patients with augmented renal clearance, standard doses of $\beta$-lactam antibiotics could yield subtherapeutic anti-infective levels.

- TDM of piperacillin-tazobactam and meropenem could contribute to optimise dosing of these antibiotics in critically ill patients.

What this study adds

- Even when dosing is assisted by TDM, a significant proportion of critically ill patients receiving $\beta$-lactams by continuous intravenous infusion could have plasma levels under their therapeutic range.

- When $\beta$-lactam TDM is carried out in patients admitted to an ICU, real-time results should be available.

There are limitations in this study. First, the twice weekly assay established in our TDM programme results in a maximum 4-day turnaround time which could be considered excessive and does not yield sufficient data to make appropriate decisions. Second, Cmax data were available in a limited number of patients, especially those with MEM. This fact could jeopardise the reliability of $\mathrm{Vd}$ values and did not permit comparisons between different groups of patients; indeed, it would have been valuable to compare the Vd values of patients with CVVHF and/or mechanical ventilation versus those without these conditions. Third, the large proportion of inadequate $\mathrm{PK} / \mathrm{PD}$ indexes observed in our study, especially for PTZ, must be considered in relation to the important proportion of patients with empirical MIC target, and may have been different with other specific/empirical treatment distributions.

\section{CONCLUSIONS}

Compliance with PD requirements in critical septic patients with preserved renal function who receive PTZ or MEM by CII assisted by TDM could be still suboptimal. The value of a TDM programme to optimise PTZ and MEM dosing in ICU patients during clinical routine practice remains controversial and could be limited if plasma levels are not available in real time. In addition, a better understanding of the potential value of TDM is needed among managers and clinicians, especially because using aggressive PD targets of $100 \% \mathrm{fT}>4-5 \times \mathrm{MIC}$ will require the administration of higher than usual recommended doses in this setting.

\section{Acknowledgements To Elena Rodríguez-Murphy (Translation).}

Contributors JAS-A designed the data collection tools, monitored data collection for the whole study, wrote and performed the statistical analysis plan, cleaned and analysed the data, and drafted and revised the paper. He is the guarantor. FA-D and MM-T implemented the study at the Pharmacy and the ICU respectively, collected the data and registered it and revised the paper. AA-E developed the analytical methods and performed the blood concentration determinations of the antibiotics. She also revised the paper. MC-M and MP-M revised the draft paper. All the authors designed the study.

Funding The authors have not declared a specific grant for this research from any funding agency in the public, commercial or not-for-profit sectors.

Competing interests None declared.

Patient consent for publication Not required. 
Provenance and peer review Not commissioned; externally peer reviewed.

ORCID iD

Joan Antoni Schoenenberger-Arnaiz http://orcid.org/0000-0002-8563-8358

\section{REFERENCES}

1 Roberts JA, Norris R, Paterson DL, et al. Therapeutic drug monitoring of antimicrobials. Br J Clin Pharmacol 2012:73:27-36.

2 Li C, Kuti JL, Nightingale CH, et al. Population pharmacokinetics and pharmacodynamics of piperacillin/tazobactam in patients with complicated intraabdominal infection. J Antimicrob Chemother 2005;56:388-95.

3 Ariano RE, Nyhlén A, Donnelly JP, et al. Pharmacokinetics and pharmacodynamics of meropenem in febrile neutropenic patients with bacteremia. Ann Pharmacother 2005:39:32-8.

4 Roberts JA, Paul SK, Akova M, et al. DALl: defining antibiotic levels in intensive care unit patients: are current $\beta$-lactam antibiotic doses sufficient for critically ill patients? Clin Infect Dis 2014:58:1072-83.

5 De Waele JJ, Lipman J, Akova M, et al. Risk factors for target non-attainment during empirical treatment with $\beta$-lactam antibiotics in critically ill patients. Intensive Care Med 2014;40:1340-51.

6 Thomas JK, Forrest A, Bhavnani SM, et al. Pharmacodynamic evaluation of factors associated with the development of bacterial resistance in acutely ill patients during therapy. Antimicrob Agents Chemother 1998:42:521-7.

7 Scheetz MH, Hurt KM, Noskin GA, et al. Applying antimicrobial pharmacodynamics to resistant gram-negative pathogens. Am J Health Syst Pharm 2006;63:1346-60.

8 Jager NGL, van Hest RM, Lipman J, et al. Therapeutic drug monitoring of anti-infective agents in critically ill patients. Expert Rev Clin Pharmacol 2016;9:961-79.

9 Wong G, Sime FB, Lipman J, et al. How do we use therapeutic drug monitoring to improve outcomes from severe infections in critically ill patients? BMC Infect Dis 2014:14.

10 Muller AE, Huttner B, Huttner A. Therapeutic drug monitoring of beta-lactams and other antibiotics in the intensive care unit: which agents, which patients and which infections? Drugs 2018;78:439-51.

11 Dulhunty JM, Roberts JA, Davis JS, et al. Continuous infusion of beta-lactam antibiotics in severe sepsis: a multicenter double-blind, randomized controlled trial. Clin Infect Dis 2013:56:236-44.

12 Falagas ME, Tansarli GS, Ikawa K, et al. Clinical outcomes with extended or continuous versus short-term intravenous infusion of carbapenems and piperacillin tazobactam: a systematic review and meta-analysis. Clin Infect Dis 2013;56:272-82.

13 Abdul-Aziz MH, Sulaiman H, Mat-Nor M-B, et al. Beta-lactam infusion in severe sepsis (bliss): a prospective, two-centre, open-labelled randomised controlled trial of continuous versus intermittent beta-lactam infusion in critically ill patients with severe sepsis. Intensive Care Med 2016;42:1535-45.

14 Perrott J, Mabasa VH, Ensom MHH. Comparing outcomes of meropenem administration strategies based on pharmacokinetic and pharmacodynamic principles: a qualitative systematic review. Ann Pharmacother 2010;44:557-64.

15 Levy MM, Fink MP, Marshall JC, et al. 2001 SCCM/ESICM/ACCP/ATS/SIS international sepsis definitions conference. Intensive Care Med 2003;29:530-8.

16 Meropenem, technical brochure AEMPS. Available: https://www.aemps.gob.es/cima/ pdfs/es/ft/73889/73889_ft.pdf [Accessed 25 Mar 2017].

17 Piperacilina-tazobactam, technical brochure AEMPS. Available: https://www.aemps. gob.es/cima/pdfs/es/ft/70433/FT_70433.pdf [Accessed 25 Mar 2017].

18 Legrand T, Chhun S, Rey E, et al. Simultaneous determination of three carbapenem antibiotics in plasma by HPLC with ultraviolet detection. I Chromatogr B Analyt Technol Biomed Life Sci 2008:875:551-6.

19 CLSI. Performance standards for antimicrobial susceptibility testing; Twenty-Fifth informational supplement. CLSI document M100-S25. Wayne, PA: Clinical and Laboratory Standards Institute, 2015.

20 Knaus WA, Draper EA, Wagner DP, et al. APACHE II: a severity of disease classification system. Crit Care Med 1985;13:818-29.

21 Levey AS, Stevens LA, Schmid CH, et al. A new equation to estimate glomerular filtration rate. Ann Intern Med 2009:150:604-12.

22 Pea F, Cojutti P, Dose L, et al. A 1 year retrospective audit of quality indicators of clinical pharmacological advice for personalized linezolid dosing: one stone for two birds? Br J Clin Pharmacol 2016;81:341-8.
23 Han Z, Pettit NN, Landon EM, et al. Impact of pharmacy practice model expansion on pharmacokinetic services: optimization of vancomycin dosing and improved patient safety. Hosp Pharm 2017;52:273-9.

24 Roberts JA, Webb S, Paterson D, et al. A systematic review on clinical benefits of continuous administration of beta-lactam antibiotics. Crit Care Med 2009;37:2071-8.

25 Sime FB, Roberts MS, Peake SL, et al. Does beta-lactam pharmacokinetic variability in critically ill patients justify therapeutic drug monitoring? A systematic review. Ann Intensive Care 2012;2.

26 Abdul-Aziz MH, Dulhunty JM, Bellomo R, et al. Continuous beta-lactam infusion in critically ill patients: the clinical evidence. Ann Intensive Care 2012;2.

27 Carrié C, Legeron R, Petit L, et al. Higher than standard dosing regimen are needed to achieve optimal antibiotic exposure in critically ill patients with augmented renal clearance receiving piperacillin-tazobactam administered by continuous infusion. J Crit Care 2018:48:66-71.

28 Aardema H, Nannan Panday P, Wessels M, et al. Target attainment with continuous dosing of piperacillin/tazobactam in critical illness: a prospective observational study. Int J Antimicrob Agents 2017:50:68-73.

29 Pea F, Della Siega P, Cojutti P, et al. Might real-time pharmacokineticl pharmacodynamic optimisation of high-dose continuous-infusion meropenem improve clinical cure in infections caused by KPC-producing Klebsiella pneumoniae? Int J Antimicrob Agents 2017;49:255-8

30 Zander J, Döbbeler G, Nagel D, et al. Piperacillin concentration in relation to therapeutic range in critically ill patients--a prospective observational study. Crit Care 2016;20.

31 De Waele JJ, Carrette S, Carlier M, et al. Therapeutic drug monitoring-based dose optimisation of piperacillin and meropenem: a randomised controlled trial. Intensive Care Med 2014;40:380-7

32 Mueller SC, Majcher-Peszynska J, Hickstein $\mathrm{H}$, et al. Pharmacokinetics of piperacillintazobactam in anuric intensive care patients during continuous venovenous hemodialysis. Antimicrob Agents Chemother 2002;46:1557-60.

33 Taccone FS, Cotton F, Roisin S, et al. Optimal meropenem concentrations to treat multidrug-resistant Pseudomonas aeruginosa septic shock. Antimicrob Agents Chemother 2012:56:2129-31.

34 Roberts JA, Aziz MHA, Lipman J, et al. Challenges and potential solutions individualised antibiotic dosing at the bedside for critically ill patients: a structured review. Lancet Infect Dis 2014;14:498-509.

35 Jacobs A, Taccone FS, Roberts JA, et al. $\beta$-Lactam dosage regimens in septic patients with augmented renal clearance. Antimicrob Agents Chemother 2018;62:e02534-17.

36 Minichmayr IK, Roberts JA, Frey OR, et al. Development of a dosing nomogram for continuous-infusion meropenem in critically ill patients based on a validated population pharmacokinetic model. J Antimicrob Chemother 2018;73:1330-9.

37 Cojutti P, Sartor A, Righi E, et al. Population pharmacokinetics of high-dose continuous-infusion meropenem and considerations for use in the treatment of infections due to KPC-producing Klebsiella pneumoniae. Antimicrob Agents Chemother 2017;61:e00794.

38 Dhaese SAM, Roberts JA, Carlier M, et al. Population pharmacokinetics of continuous infusion of piperacillin in critically ill patients. Int J Antimicrob Agents 2018:51:594-600.

39 Maglio D, Teng R, Thyrum PT, et al. Pharmacokinetic profile of meropenem, administered at 500 milligrams every 8 hours, in plasma and cantharidin-induced skin blister fluid. Antimicrob Agents Chemother 2003;47:1771-3.

40 Rhodes NJ, MacVane SH, Kuti JL, et al. Impact of loading doses on the time to adequate predicted beta-lactam concentrations in prolonged and continuous infusion dosing schemes. Clin Infect Dis 2014;59:905-7.

41 Seyler L, Cotton F, Taccone FS, et al. Recommended $\beta$-lactam regimens are inadequate in septic patients treated with continuous renal replacement therapy. Crit Care 2011;15.

42 Jaruratanasirikul S, Sriwiriyajan S, Punyo J. Comparison of the pharmacodynamics of meropenem in patients with ventilator-associated pneumonia following administration by 3-hour infusion or bolus injection. Antimicrob Agents Chemother 2005:49:1337-9.

43 Isla A, Rodríguez-Gascón A, Trocóniz IF, et al. Population pharmacokinetics of meropenem in critically ill patients undergoing continuous renal replacement therapy. Clin Pharmacokinet 2008:47:173-80.

44 Shekar K, Fraser JF, Taccone FS, et al. The combined effects of extracorporeal membrane oxygenation and renal replacement therapy on meropenem pharmacokinetics: a matched cohort study. Critical Care 2014;18. 